\title{
Por una filosofía crítica de la historia *
}

\author{
JOHANNES ROHBECK \\ Technische Universität, Dresden
}

\begin{abstract}
RESUMEN: Este artículo propone una renovación de la filosofía de la historia que tenga en cuenta los resultados del giro hacia la teoría de la cultura y hacia el concepto de espacio. Esta filosofía de la historia es material porque no se limita a la metodología y es crítica porque intenta proporcionar una orientación ética para la vida, una reflexión sobre las posibilidades reales de actuar.
\end{abstract}

Palabras clave: Filosofía de la historia, Teoría de la cultura, Ética.
ABSTRACT: This paper propounds a renewal of philosophy of history that takes account of the outcomes of both the «spatial turn» and the turn towards theory of culture. This philosophy of history is intended as a material one because it goes beyond methodology, and as a critical one because it tries to furnish an ethical orientation for life, a reflection on the real possibilities to act.

Key words: Philosophy of history, Culture theory, Ethics.

\section{Crítica de la filosofía de la historia}

Hoy parece ser un lugar común que la filosofía de la historia ha fracasado. Es inevitable renunciar al proyecto de interpretar la historia de toda la humanidad como un nexo de sentido, un proyecto que se plasmó en las ideas de la Ilustración sobre el «progreso continuo hacia lo mejor» y que a continuación fue modificado por la concepción hegeliana del «espíritu del mundo» y la «astucia de la razón» y por la reinterpretación materialista de Marx. Si el concepto «filosofía de la historia» se refiere a este proyecto, la crítica parece necesaria; tenemos que preguntarnos si este acceso a la historia no ha abandonado desde el principio el ámbito de los problemas filosóficos legítimos y ha rebasado los límites de una teoría filosófica argumentativa.

La crítica que plantea esta objeción ha sido formulada muchas veces, en especial por los autores y autoras que derivan las catástrofes políticas del siglo XX en parte de la fe doctrinaria en el progreso. En detalle, esta crítica presenta muchos matices diferentes ${ }^{1}$.

* Traducción de Christian Baumann, con la colaboración de Jorge Navarro Pérez.

${ }^{1}$ Cfr. Herta Nagl-Docekal: «Ist Geschichtsphilosophie heute noch möglich?», en H. N.-D. (ed.), Der Sinn des Historischen. Geschichtsphilosophische Debatten, Fráncfort, 1996, 7-66; Johannes Rohbeck, Geschichtsphilosophie zur Einführung, Hamburgo, 2003. 
Recordemos algunos. Desde el punto de vista de la teoría del conocimiento, la filosofía analítica plantea la objeción de que una tesis sobre «toda» la evolución de la humanidad tiene que ver también con el futuro, por lo que forma parte del ámbito de la profecía, que no tiene carácter científico (Danto ${ }^{2}$. Representantes de la Teoría Crítica como Benjamin, Adorno y Horkheimer censuran una toma de partido que tiene graves consecuencias: entender la historia como un progreso continuo equivale a hacerse cómplice de los vencedores, lo cual impide ver bien a las víctimas de los conflictos del pasado ${ }^{3}$. Para Karl Löwith, la filosofía de la historia se basa en una secularización problemática de la historia sagrada ${ }^{4}$. Por último, desde el punto de vista del pensamiento postmoderno Lyotard interpretó la filosofía de la historia como el «gran relato» que le sirve de «relato de legitimación» al «terror de la homogeneización» que caracteriza a la modernidad ${ }^{5}$.

Por supuesto, estas críticas no implican que la historia haya de quedar fuera del ámbito de los temas filosóficos legítimos. Hoy también se buscan las posibilidades de una «filosofía de la historia tras el final de la filosofía clásica de la historia» ${ }^{6}$. Como se sabe, el rechazo del proyecto inicial ha sacado a la luz otros problemas, de modo que el concepto «filosofía de la historia» ha sido definido de nuevas maneras.

Mientras que las afirmaciones sobre la historia son tildadas de «sustancialistas» y resultan sospechosas de ideología, el interés se traslada a los métodos de las ciencias de la historia. A lo largo del tiempo, este programa científico ha variado entre «comprender» y «explicar» los acontecimientos históricos, así como «valorar» acciones con relevancia histórica 7 . Como consecuencia del linguistic turn, estas investigaciones culminan en el análisis del lenguaje y del discurso, en la semiótica de los conceptos de tiempo ${ }^{8}$ y en el

${ }^{2}$ Karl Popper, «Prediction and Prophecy in the Social Sciences», en Patrick Gardiner (ed.), Theories of History, Glencoe, 1959, 276-284; Arthur C. Danto, Analytische Philosophie der Geschichte, Fráncfort, 1974, 11-35.

3 Walter Benjamin, «Über den Begriff der Geschichte», en W. B., Illuminationen. Ausgewählte Schriften, Fráncfort, 1977, 251-261; Max Horkheimer y Theodor W. Adorno, Dialektik der Aufklärung, Fráncfort, 1969.

${ }^{4}$ Karl Löwith, Weltgeschichte und Heilsgeschehen, Stuttgart, 1953.

5 Jean-François Lyotard, «Das postmoderne Wissen», en Theatro machinarum, n. ${ }^{\circ} 3 / 4$, $1982,59-70$.

${ }^{6}$ Hans-Michael Baumgartner, «Philosophie der Geschichte nach dem Ende der Geschichtsphilosophie. Bemerkungen zum gegenwärtigen Stand geschichtsphilosophischen Denkens», en H. Nagl-Docekal (ed.), Der Sinn des Historischen, Fráncfort, 1996, 151-172.

7 Wilhelm Dilthey, Der Aufbau der geschichtlichen Welt in den Geisteswissenschaften, ed. M. Riedel, Fráncfort, 1970; Johann Gustav Droysen, Historik. Textausgabe von Peter Leyh, Stuttgart, 1977; Ernst Troeltsch, Schriften zur Politik und Kulturphilosophie (1918-1923), ed. Gangolf Hübinger, Berlín y Nueva York, 2002; Georg Henrik von Wright, Erklären und Verstehen, Königstein, 1974.

8 Reinhart Koselleck, Vergangene Zukunft. Zur Semantik geschichtlicher Zeiten, Fráncfort, 1979; Johannes Rohbeck, «Verzeitlichung», en Historisches Wörterbuch der Philosophie, ed. Joachim Ritter y Karlfried Gründer, vol. 11, Basilea, 2001, 1026 ss. 
análisis de las estructuras narrativas, es decir, literario-históricas ${ }^{9}$. El complemento de estas investigaciones son los análisis del modo del recuerdo histórico, de las culturas del recuerdo contemporáneas y pasadas. Aquí tiene su lugar también el tema de la identidad histórica: nacional, intercultural, tal vez incluso global. Este tema es importante para la «identidad europea».

\section{Filosofía de la historia y crítica de la cultura}

No se pueden negar los resultados de estos análisis. Pero me gustaría plantear unas preguntas ${ }^{10}$ : ¿es obligatorio reducir la filosofía de la historia a los temas metodológicos? ¿Es posible una crítica de la filosofía inicial de la historia que la ponga a salvo? ¿Es posible una filosofía que aborde la cuestión metodológica y al mismo tiempo sea material?

Desde hace algún tiempo se habla de un giro hacia las ciencias de la cultura. Quiero discutir aquí las consecuencias que este cambio de paradigma puede tener para la filosofía de la historia ${ }^{11}$. También quiero averiguar de qué manera se puede conectar la filosofía de la historia con la teoría de la cultura.

El concepto de cultura es hoy relativamente vago. Por una parte, las ciencias de la cultura utilizan métodos ya acreditados, como los de la semiótica y de la teoría del discurso. De acuerdo con esto, la «cultura» es un sistema de signos que interpreta la realidad con sentido para unos grupos sociales determinados; aquí desempeñan una función decisiva las formas simbólicas de representación. Pero por otra parte en los recientes cultural studies se han producido unos cambios que también podrían ser importantes para la filosofía de la historia: estudiar los fenómenos del mundo de la vida, integrar la historia de las ciencias y la historia social en la «historia del espíritu», así como salvar la distancia entre las «dos culturas», entre las ciencias de la naturaleza y las ciencias del espíritu, entre el conocimiento y la estética, entre la escritura y la imagen, etc. ${ }^{12}$ De este modo se presentan posibilidades de superar la reducción de la filosofía de la historia a la metodología y de ampliar su temática.

\footnotetext{
${ }^{9}$ Cfr. Hayden White, Die Bedeutung der Form. Erzählstrukturen in der Geschichtsschreibung, Fráncfort, 1990; Metahistory. Die historische Einbildungskraft im 19. Jahrhundert in Europa, Fráncfort, 1991; Frank Ankersmit y Hans Kellner (eds.), A New Philosophy of History, Chicago y Londres, 1995; Paul Ricoeur, Zeit und Erzählung, 3 vols., Múnich, 1988-1991.

10 Johannes Rohbeck, Technik - Kultur - Geschichte. Eine Rehabilitierung der Geschichtsphilosophie, Fráncfort, 2000, 42 ss.

11 Ralf Konersmann (ed.), Kulturphilosophie, Leipzig, 1996; Adam Kuper, Culture. The Anthopologist's Account, Cambridge y Londres, 1999; Hartmut Böhme, Peter Matussek y Lothar Müller, Orientierung Kulturwissenschaft, Reinbek, 2000.

12 Charles P. Snow, Die zwei Kulturen. Literarische und naturwissenschaftliche Intelligenz, Stuttgart, 1967; Georg Bollenbeck, Bildung und Kultur. Glanz und Elend eines deutschen Deutungsmusters, Fráncfort y Leipzig, 1994.
} 
También se ofrece la oportunidad de conectar de una manera novedosa con las tradiciones de la filosofía de la historia. Aunque ya no compartamos la idea ilustrada de progreso, podemos admitir que los filósofos de la historia del siglo XVIII escribieron historias de la cultura en el sentido estricto ${ }^{13}$. Autores como Voltaire y Turgot, así como Ferguson, Schlözer y Herder, no sólo descubrieron culturas antiguas y no europeas, sino que además desarrollaron una concepción compleja al entender por «cultura» la conexión que se da en cada caso entre la ciencia, la técnica, la economía, la política y la moral ${ }^{14}$. Hoy en día, un proyecto reformulado de filosofía de la historia puede conectar con este tipo de historia de la cultura.

También en el historicismo sobrevivió, junto a la metodología, esta idea de cultura, pero con un menosprecio claro de la civilización científico-técnica y una sobrevaloración de la cultura puramente «espiritual» de lo político y lo religioso. Aquí tiene sus raíces la funesta separación entre una «civilización» presuntamente ahistórica y una «cultura» histórica. El historicismo entiende por cultura ante todo las «potencias éticas» o los valores morales ${ }^{15}$. Sin embargo, este planteamiento podría ser importante para una filosofía actual de la historia, sobre todo porque intenta elaborar una fundamentación normativa de lo histórico.

La relación entre historia y cultura se revela ambivalente en la filosofía de la posthistoire ${ }^{16}$. Por una parte interpreta el proceso de la civilización como un «final de la historia» porque las sociedades modernas de masas, con su primacía de la técnica y el consumo, desplazan a culturas que han crecido con la historia, pero no son capaces de producir ofertas culturales de sentido. Por otra parte, la posthistoire tematiza las nuevas tecnologías y las analiza en relación con sus efectos culturales negativos. Un trato diferenciado con esta po-

13 Cfr. Wilhelm Dilthey, Das 18. Jahrhundert und die geschichtliche Welt, en Gesammelte Schriften, vol. 3, Leipzig, 1927, 235 ss.; Emil Spiess, «Der frühe Versuch einer allgemeinen Kulturgeschichte auf evolutionistischer Grundlage», en Studien aus dem Gebiet von Kirche und Kultur. Festschrift für Gustav Schnürer, 1930; Kurt Breysig, Die Meister der entwickelnden Geschichtsforschung, Bresláu, 1936.

${ }_{14}$ Voltaire, Essai sur l'histoire générale et sur les moeurs et l'esprit des nations, 7 vols., Ginebra, 1756; Anne Robert Jacques Turgot, Über die Fortschritte des menschlichen Geistes, ed. Johannes Rohbeck y Lieselotte Steinbrügge, Fráncfort, 1990; Adam Ferguson, Versuch über die Geschichte der bürgerlichen Gesellschaft, ed. Zwi Batscha y Hans Medick, Fráncfort, 1988; Johann Gottfried Herder, Auch eine Philosophie der Geschichte zur Bildung der Menschheit, epílogo de Hans-Georg Gadamer, Fráncfort, 1967; August Wilhelm von Schlözer, Universalhistorie, Gotinga, 1772.

15 Wilhelm Dilthey, Der Aufbau der geschichtlichen Welt in den Geisteswissenschaften, ed. M. Riedel, Fráncfort, 1970; Johann Gustav Droysen, Historik. Textausgabe von Peter Leyh, Stuttgart, 1977.

16 Jean-François Lyotard, «Das postmoderne Wissen», en Theatro machinarum, n. ${ }^{\circ} 3 / 4$, 1982; Jean Baudrillard, Der symbolische Tausch und der Tod, Múnich, 1982; Vilém Flusser, Nachgeschichte. Eine korrigierte Geschichtsschreibung, ed. Stefan Bollmann y Edith Flusser, Fráncfort, 1997; Paul Virilio, Rasender Stillstand, Fráncfort, 1997; José Ortega y Gasset, La rebelión de las masas, Madrid, 1930. 
sición podría integrar las observaciones críticas en una filosofía cultural de la historia, evitando las conclusiones «postmodernas».

En este contexto aparece el concepto de «crítica de la cultura» ${ }^{17}$. Investigaciones recientes han sacado a la luz una serie de similitudes entre la Teoría Crítica y la teoría de la postmodernidad, entre la Escuela de Fráncfort y el postestructuralismo francés (Breitenstein). Estas similitudes se muestran en la tematización de las experiencias de alienación, así como en las propuestas de una filosofía negativa de la historia. Aunque los pronósticos apocalípticos de la vieja crítica de la cultura no se han confirmado, una filosofía de la historia guiada por la teoría de la cultura ha de adoptar una actitud «crítica» ante el transcurso fáctico de la historia.

\section{Normatividad y facticidad de lo histórico}

Aquí se abren varias posibilidades que voy a dividir, como si fueran tipos ideales, en dos corrientes. Con esta alternativa intento caracterizar el debate actual en Alemania sobre si la filosofía de la historia ha de tener una orientación material o ha de basarse en una filosofía de la cultura. Una de estas dos corrientes prosigue la tradición de Immanuel Kant; la otra, la tradición de Hegel y Marx. Así pues, aquí se repite la oposición «clásica» entre Kant y Hegel.

La primera alternativa de una filosofía de la historia basada en la crítica de la cultura se refiere al nivel de la ética ${ }^{18}$. Sus representantes (Nagl-Docekal, Gröbler-Steinbach, Lutz-Bachmann) reprochan con razón a la crítica habitual de la concepción inicial de la filosofía de la historia que no haya tomado en cuenta que la idea del «progreso hacia lo mejor» no fue formulada en sentido empírico-descriptivo, sino con una intención moral. En este punto comienza la filosofía de la historia de Kant, que intenta al menos abrir una perspectiva posible de esperanza, sin la cual sería absurdo esforzarse por incrementar la moralidad. Kant no elabora un «gran relato», sino que analiza la

17 Theodor W. Adorno, «Kulturkritik und Gesellschaft», en: id., Prismen. Ohne Leitbild, Gesammelte Schriften, vol. 10/1, Fráncfort, 1977, 11 ss.; Ralf Konersmann (ed.), Kulturkritik, Leipzig, 2001; Johannes Rohbeck y Herta Nagl-Docekal (eds.), Geschichtsphilosophie und Kulturkritik. Historische und systematische Studien, Darmstadt, 2003.

18 Evelyn Gröbl-Steinbach, Fortschrittsidee und rationale Weltgestaltung. Die kulturellen Voraussetzungen des Politischen in der Moderne, Fráncfort y Nueva York, 1994; "Geschichtsphilosophie und postmoderne Vernunftkritik», en Rohbeck y Nagl-Docekal (eds.), op. cit., 136 ss.; Herta Nagl-Docekal, «Hoffen auf künftige Freiheit. Führt Hannah Arendts Konzeption des Politischen die Geschichtsphilosophie Kants weiter?» en Rohbeck y Nagl-Docekal (eds.), op. cit., 231 ss.; M. Lutz-Bachmann, Geschichte und Subjekt. Zum Begriff der Geschichtsphilosophie bei Immanuel Kant und Karl Marx, Friburgo y Múnich, 1988; «Subjekt und Geschichte. Über die Aufgaben der Geschichtsphilosophie heute», en Rohbeck y Nagl-Docekal (eds.), op. cit., 231 ss. 
cuestión de si la lucha por la justicia y la paz que estamos obligados moralmente a entablar tiene posibilidades de éxito a la vista del curso violento que hasta ahora han tomado las cosas.

La actualidad de esta postura consiste en que las crisis y los conflictos actuales, así como las experiencias de injusticia y culpa, vuelven virulenta la cuestión del sentido. Mediante estas reclamaciones de sentido se intenta mitigar la circunstancia de que no podemos disponer de la historia. Y esto se basa en la convicción de que el tratamiento filosófico de este tipo de preguntas consigue ir, también hoy, más allá de lo presente y real.

La segunda alternativa consiste en una filosofía material de la historia que no se limita a la normatividad de la historia, sino que incluye su facticidad. La referencia a Kant contenía la paradoja de que se adaptaba a Kant porque no escribió una filosofía de la historia, sino que observó la historia desde fuera con los medios de la razón práctica.

Pero la conexión de filosofía de la historia y ética no nos obliga en absoluto a despedirnos del «gran relato». La objeción de que la filosofía de la historia se puso en el lugar de la filosofía práctica, privando a ésta de sus potenciales críticos, no es válida para el pensamiento histórico de la Ilustración ${ }^{19}$. Tampoco es válido para el presente el reproche de que la filosofía de la historia sustituyó las pretensiones normativas de validez por la facticidad de los procesos históricos. Al contrario, el estudio de la historia nos permite averiguar qué metas no se realizaron en el pasado, son realizables en el presente y se deberían realizar en el futuro.

A la inversa, una ética que menosprecia los desarrollos históricos pierde la referencia a la realidad. Así como debemos evitar el peligro del naturalismo, que intenta erróneamente elaborar una justificación moral a partir del transcurso fáctico de la historia, también existe el peligro contrario del normativismo, que consiste en establecer unas normas ajenas a la realidad. Algunos éticos no pueden resistirse a la tentación de transformar el mundo de acuerdo con principios abstractos. No es de extrañar que tras tantos esfuerzos sin éxito reine la decepción. A diferencia de la ética, la filosofía de la historia no trata de la fundamentación de las metas morales, sino de su realización a largo plazo. La filosofía de la historia representa, frente a la ética, el principio de realidad.

19 Jürgen Habermas, Der philosophische Diskurs der Moderne, Fráncfort, 1985, 9 ss.; Faktizität und Geltung. Beiträge zur Diskurstheorie des Rechts und des demokratischen Rechtsstaats, Fráncfort, 1994, 16-17; Herbert Schnädelbach, Geschichtsphilosophie nach Hegel. Die Probleme des Historismus, Friburgo y Múnich, 1974, 41-42; Wolfgang Bialas, Von der Revolution der Klasse zur Evolution der Vernunft. Vernunftphilosophie in kommunikationstheoretischer Begründung, Fráncfort, 1996, 109. 


\section{Filosofía crítica de la historia}

En este punto comienzan mis reflexiones para una rehabilitación de la filosofía de la historia y una «filosofía crítica de la historia». Siguiendo a otros filósofos ilustrados, como Turgot, Condorcet, Ferguson y Schlözer, así como a Hegel y Marx, es posible reflexionar mediante la filosofía de la historia sobre el transcurso fáctico de los procesos históricos. Sin duda, procesos actuales como la tendencia a la globalización o la modernización progresiva poseen también una dimensión genuinamente histórica; por tanto, podemos tematizarlos filosóficamente.

Así, el proceso de la civilización moderna presenta una continuidad asombrosa ${ }^{20}$. Lo que la Ilustración imaginó ya es real: el desarrollo científico, técnico y económico ha resistido al ascenso y descenso de las naciones, incluso durante las catástrofes del siglo XX. Las expectativas formuladas en las primeras historias universales han sido superadas ampliamente en este campo. Ningún otro ámbito de la vida humana muestra tanta homogeneidad. Yo defiendo la tesis de una radicalización de la modernidad porque veo en los cambios actuales la continuidad de tendencias de desarrollo típicamente modernas. Esta tesis incluye explícitamente la pluralidad de las culturas y, por tanto, también de las modernidades diferentes culturalmente. Cuando últimamente se habla de las modernidades europea, americana y japonesa, se presupone al mismo tiempo un tipo común de la modernidad en aceleración.

Pienso que también tiene sentido actualizar la idea de historia universal o mundial. Cuando a finales del siglo XVIII los viajes de descubrimiento se acabaron porque la geografía no daba más de sí, surgieron por primera vez las comparaciones universales y sistemáticas de culturas. Esta conexión entre la desaparición de los límites y la delimitación hizo posible la filosofía de la historia. Hoy conocemos como «globalización» el hecho de que el nexo práctico de acción se ha vuelto global y, por tanto, la historia mundial se ha vuelto real. La historia universal ya no significa el desfile triunfal de la civilización europea, sino la integración de las diversas culturas dentro de un sistema de cooperación nuevo. Así, los historiadores ven en la historia universal un enfoque legítimo y particular cuyo objeto son los nexos de acción que se extienden por espacios amplios ${ }^{21}$. Esto debería animar a los filósofos a reflexionar de nuevo sobre la historia en conjunto.

Estas posibilidades de actualizar las dimensiones diacrónica y sincrónica de la idea de historia mundial demuestran que aludiendo a la teleología de la historia no se despacha la filosofía de la historia. En primer lugar, porque la

\footnotetext{
20 Johannes Rohbeck, Technik - Kultur - Geschichte. Eine Rehabilitierung der Geschichtsphilosophie, Fráncfort, 2000, 212 ss.

21 Ernst Schulin (ed.), Universalgeschichte, Colonia, Kiepenheuer und Witsch, 1974; Jürgen Osterhammel, Geschichtswissenschaft jenseits des Nationalstaates, Gotinga, Vandenhoeck und Ruprecht, 2001.
} 
filosofía de la historia no se reduce a la teleología, como muestran sus componentes histórico-estructurales y explicativos. En segundo lugar, porque la teleología contiene ciertos conocimientos que vale la pena reformular.

Esto se refiere en primer lugar a la experiencia histórica de la contingencia $^{22}$, gracias a la cual sabemos que la historia se sustrae a la planificación humana. Los seres humanos no pueden prever qué consecuencias tendrán sus acciones dentro del proceso histórico. Tras los horrores del siglo Xx y la reciente guerra en Iraq se impone la conclusión de que tenemos que despedirnos de la locura de que podemos hacer la historia a nuestro gusto.

Pero esta posición mínima se releva insuficiente cuando situamos la filosofía de la historia en el contexto de la filosofía práctica. Desde el punto de vista filosófico se espera una orientación para la acción que no se obtiene al decir que la historia no está a nuestra disposición. Aunque en conjunto la historia no es planificable, en cada momento se abren unos espacios limitados para intervenir en el curso de la historia. Para esto hacen falta unos criterios morales que orienten el comportamiento práctico de las personas. Como hemos visto, esta dimensión normativa de la filosofía de la historia se identifica ante todo con la teleología, que expone la meta de acuerdo con la cual hay que juzgar los acontecimientos del pasado y orientar las acciones del futuro.

La tarea de esta «filosofía crítica de la historia» consiste en sacar a la luz los impagos de la historia para poder cambiar las condiciones actuales de vida de acuerdo con criterios éticos ${ }^{23}$. Para hacer esto hay que poner en cuestión la presunta linealidad de la historia y su presunto final, y entonces podremos pensar la historia como un proceso más variado y abierto. Esto no significa abandonar la idea de continuidad histórica. No debemos imaginarnos la historia como un progreso lineal, pero sí como un nexo de desarrollo en el que se pueden concebir y realizar alternativas. La cuestión no es reventar la continuidad en tanto que tal, sino trazar líneas alternativas de tradición. Esta reflexión sobre las posibilidades utilizadas y desaprovechadas puede servir para mejorar la sensibilidad hacia las alternativas de acción.

\section{La «adecuación» como categoría de la filosofía de la historia}

Con estos presupuestos, el desarrollo de la civilización técnica es un tema central de la filosofía de la historia. Las técnicas nuevas no significan una pérdida total de la cultura y la historia, sino que abren horizontes nuevos para posibilidades de acción no previstas y no buscadas. Los nuevos medios técni-

22 Reinhart Koselleck, Vergangene Zukunft. Zur Semantik geschichtlicher Zeiten, Fráncfort, 1979, 260 ss.; Heinz-Dieter Kittsteiner, Out of Control. Über die Unverfügbarkeit des historischen Prozesses, Berlín y Viena, 2004, 10 ss.

23 Walter Benjamin, «Über den Begriff der Geschichte», en Gesammelte Schriften, ed. Rolf Tiedemann y Hermann Schweppenhäuser, vol. I/2, Fráncfort, 1974, 691 ss. 
cos amplían las posibilidades de uso y, por consiguiente, las condiciones para un uso social diferente de la técnica.

Voy a explicar esto recurriendo brevemente a la filosofía de la historia de Marx. En el prólogo de la Crítica de la economía política (1859), Marx resume con estas palabras la dinámica de las formaciones históricas: «En la producción social de su vida, las personas establecen unas relaciones determinadas, necesarias e independientes de su voluntad, unas relaciones de producción que corresponden a un nivel determinado de desarrollo de sus fuerzas productivas materiales. [...] En cierto nivel de su desarrollo, las fuerzas materiales de producción de la sociedad entran en contradicción con las relaciones de producción existentes» ${ }^{24}$. Las categorías operacionales importantes son «corresponder» y «contradicción». En este lugar son interesantes las investigaciones recientes del Marxismo Analítico (Cohen, Iorio), que voy a tomar en consideración sin renunciar al método dialéctico ${ }^{25}$.

El verbo «corresponder» indica una analogía que no contiene una explicación causal, pues la relación entre fuerzas productivas y relaciones de producción se puede invertir. Más adelante, el uso de los verbos «causar» y «determinar» indicará una dependencia unívoca. Marx ofrece, en vez de esto, explicaciones funcionales, es decir, unas explicaciones que indican los factores que en un sistema social desempeñan una función determinada sin ser de naturaleza teleológica. En este caso se trata de unas relaciones sociales determinadas que favorecen, estabilizan u obstaculizan el desarrollo de las tecnologías.

Si prescindimos de la retórica de una necesidad presuntamente histórica, podemos interpretar de una manera más débil la tesis central sobre la relación entre las fuerzas productivas y las relaciones de producción. Las categorías mencionadas de «corresponder» y «contradecir» se pueden interpretar también de esta manera: se afirma simplemente que los nuevos medios técnicos crean posibilidades ampliadas de uso y, por tanto, los presupuestos para otro uso social de la técnica. Son posibilidades reales por las que la sociedad se puede orientar. A la inversa, se trata simplemente de posibilidades y, sobre todo, de varias alternativas cuya realización no está determinada en absoluto por la técnica.

Para caracterizar a este margen de maniobra entre la determinación y la apertura de horizonte, utilizo el concepto de adecuación como categoría de la filosofía de la historia ${ }^{26}$. Este concepto se refiere a la relación cambiante entre las condiciones fácticas y las ideas normativas, en este caso entre los medios técnicos y las metas justificadas éticamente.

${ }^{24}$ Karl Marx y Friedrich Engels, Marx Engels Werke, Berlín, 1956 ss., vol. 13, 8-9.

25 Gerald A. Cohen, Marx' Theory of History. A Defense, Oxford, Clarendon Press, 1978; Marco Iorio, Karl Marx - Geschichte, Gesellschaft, Politik. Eine Ein- und Weiterführung, Berlin, 2003; Johannes Rohbeck, Marx, Stuttgart, 2006, 86 ss., 97 ss.

26 Johannes Rohbeck, Geschichtsphilosophie zur Einführung, Hamburgo, 2003, 164 ss. 
Por una parte, las metas dirigen nuestra actuación; se convierten en fines prácticos cuando se realizan con ayuda de unos medios determinados. Estos medios son adecuados si cumplen la función de realizar el fin; por tanto «adecuado» significa funcional, útil o provechoso. En su contexto histórico, adecuación significa que, una vez reconocidas ciertas metas, hay que emplear o crear los medios necesarios para alcanzarlas. Así pues, el establecimiento dinámico de metas sirve de motor del desarrollo técnico. Cuando las metas se amplían, los viejos medios ya no se consideran adecuados y son sustituidos por nuevas técnicas.

Por otra parte, los medios amplían el horizonte de acciones posibles y el campo para establecer metas. La relación inversa entre fin y medio también es muy influyente históricamente. Este efecto retroactivo fue descubierto por muchos filósofos de la historia: por los representantes de la Ilustración hasta Marx, incluso por Burckhardt. Esta inversión tiene una consecuencia para la categoría de adecuación: también los fines han de ser adecuados a los medios técnicos. Pues cuando surge una nueva meta de acción, que ya es alcanzable técnicamente, surge también la expectativa de realizar esta posibilidad.

No hay que confundir este tipo de adecuación con la adaptación ciega al progreso técnico. Lo principal son las metas legitimadas por la ética. En general se habla de la idea de la vida buena o de valores como bienestar, seguridad, libertad, salud, etc. A esto hay que añadir valores como movilidad y comunicación, los cuales han sido reforzados o incluso creados por la civilización moderna. Pero cómo hay que entender esto en unas situaciones determinadas y cómo hay que convertir estas metas generales en fines concretos de acción depende también de las posibilidades de realización. En cada caso hay que examinar exactamente en qué medida los fines así modificados son adecuados no sólo para las posibilidades técnicas, sino también para los valores asumibles social y ecológicamente.

Como consecuencia de la globalización, la desigualdad de riqueza entre los diversos países y regiones es cada vez más evidente. Desde la perspectiva histórica, este fenómeno se basa en desarrollos «no-simultáneos» que han conducido a niveles diferentes de la civilización moderna. También en el caso de la comparabilidad global se puede hablar de adecuación o inadecuación. Cuando la población de un país se da cuenta de que en otro país se vive mejor, puede empezar a desear vivir así (cfr. Burgio). Mediante la comparación las personas obtienen no sólo criterios nuevos para juzgar su propia civilización, sino también criterios para realizar unos deseos que albergan desde hace mucho tiempo. De este modo, el concepto de no-simultaneidad de la filosofía de la historia se convierte en una categoría crítica.

Así pues, un estado social es adecuado si un nivel determinado de desarrollo científico-técnico y económico hace posible vivir de una manera determinada en un nivel determinado de la cultura. A esto se suma para el futuro la expectativa de realizar nuevas posibilidades. De aquí se desprenden en los 
contextos políticos unas exigencias que intentan llevar a la práctica lo que es posible desde los puntos de vista técnico y económico.

\section{Filosofía del espacio histórico}

Durante los últimos años ha crecido el interés por el fenómeno del espacio. En general se habla del spatial turn ${ }^{27}$, y en el área de la historia se habla de spacing history ${ }^{28}$. Se trata de una conexión de espacio y tiempo, de geografía e historia: la narración histórica se renueva mediante una percepción reforzada del espacio. Se da a entender que la espacialización de la historia puede contribuir a la reformulación del pensamiento histórico. En este sentido, se ha producido un retorno del espacio histórico.

La actualidad del espacio está relacionada claramente con la globalización ${ }^{29}$. Con el nuevo orden mundial que se está perfilando ha surgido un espacio que requiere una reflexión teórica nueva. Una vez acabado el mundo bipolar se está formando una consciencia de la historia particular de los lugares. Por una parte surgen mercados abiertos y redes globales; por otra parte la gente vuelve a interesarse por lo local y regional. Se redefinen así los centros, las periferias y las fronteras. Éstas ya no transcurren entre los Estados nacionales, sino por dentro de ellos. Las ciudades son centros transterritoriales de la economía, la política y la información, funcionan como puntos de intersección en el enrejado mundial.

La globalización no «destruye» el espacio ${ }^{30}$, sino que modifica las estructuras espaciales. El hecho de que espacios lejanos parezcan «más cercanos» se debe a que las acciones humanas repercuten cada vez más lejos. Mediante el comercio, la división del trabajo y las telecomunicaciones, los espacios de acción de las personas se expanden y condensan. Algunas acciones ya no están ligadas a un lugar determinado, sino que cada espacio puede

27 En la sociología: Henri Lefebvre, The Production of Space, Oxford y Cambridge, Blackwell, 1991 (La production de l'espace, 1974); Anthony Giddens, Die Konstitution der Gesellschaft, Fráncfort, 1988.

28 Edward Soja, Postmodern Geographies. The Reassertation of Space in Critical Social Theory, Londres, 1989, 1-2; Christoph Conrad (ed.), «Mental Maps», en Geschichte und Gesellschaft. Zeitschrift für Historische Sozialwissenschaft 28 (2002), n. ${ }^{\circ}$ 3; cfr. Jürgen Osterhammel, «Die Wiederkehr des Raumes: Geopolitik, Geohistorie und historische Geographie», en Neue Politische Literatur 43 (1998), 374 ss.; Rudolf Maresch y Niels Werber (eds.), Raum Wissen - Macht, Fráncfort, 2002, 7 ss.; Karl Schlögel, Im Raume lesen wir die Zeit. Über Zivilisationsgeschichte und Geopolitik, Múnich y Viena, 2003, 60 ss.

29 Peter Noller, «Globalisierung, Raum und Gesellschaft. Elemente einer modernen Soziologie des Raumes», en Berliner Journal für Soziologie 10 (2000), n. ${ }^{\circ} 1,21$ ss.; Saskia Sassen, Machtbeben. Wohin führt die Globalisierung?, Stuttgart y Múnich, 2000, 173 ss.

${ }_{30}$ Marshall McLuhan, Die magischen Kanäle. Understanding Media, Dresde y Basilea, 1995, 149; Paul Virilio, Rasender Stillstand, Fráncfort, 1997, 126 ss. 
cumplir funciones muy variadas. De este modo, el espacio social se desacopla del espacio geométrico; la proximidad social y la proximidad geográfica se separan. Lo mismo se puede decir del espacio de la historia: al desprenderse el tiempo histórico del tiempo natural, surge un espacio específicamente histórico, que es diferente del espacio natural.

A la vista de estas reflexiones tenemos que preguntar qué consecuencias tiene el «giro hacia el espacio» para la filosofía de la historia, cómo podemos no sólo describir ese espacio historiográficamente, sino además investigarlo teóricamente, cómo debemos entender filosóficamente la espacialización de la historia.

Ya en la filosofía ilustrada de la historia se observa la conexión entre tiempo y espacio. Pues la idea de historia universal significa que todos los tiempos históricos, desde el principio hasta el presente, y todos los espacios históricos del planeta son incluidos en el estudio de la historia ${ }^{31}$. Las ampliaciones temporales y espaciales, que parecen simplemente exteriores, nos conducen a conclusiones teóricas muy importantes. Pues al comparar desde la perspectiva del progreso pueblos que viven en regiones y épocas diferentes hacemos el descubrimiento revolucionario de que niveles culturales diferentes no sólo se siguen temporalmente en el mismo lugar, sino que además se dan al mismo tiempo en lugares diferentes ${ }^{32}$. Esto es lo que más adelante se denominará simultaneidad de lo no-simultáneo [Gleichzeitigkeit des Ungleichzeitigen] ${ }^{33}$.

La comparación con la filosofía ilustrada de la historia es esclarecedora también para el pensamiento histórico contemporáneo. La simultaneidad y la no-simultaneidad se revelan el resultado de un desarrollo que empezó al principio de la Edad Moderna y que siempre ha formado parte del proyecto de la modernidad. Pese a la continuidad, que considero importante resaltar, no se puede negar que la simultaneidad producida por los medios de comunicación es de un tipo nuevo. En el siglo XVIII la simultaneidad se refería a culturas no-simultáneas en lugares diferentes de la Tierra, lejanos unos de otros, por lo que esas culturas eran estudiadas con un retraso temporal considerable. Todavía durante la época de la Ilustración se consideraba que las culturas son homogéneas y que sólo al compararlas entre sí se revelan no simultáneas.

31 Charles-Louis de Montesquieu, L'esprit des lois (1734); Nicolas Fréret, De l'antiquité et de la certitude de la chronologie chinoise (1731); Voltaire, Essai sur les moeurs, vols. XI-XIII; Guillaume Thomas François Raynal y Denis Diderot, Histoire philosophique et politique des établissements et du commerce des Européens dans les deux Indes (1770); William Robertson, The History of the Discovery and Settlement of America (1777); cfr. Johannes Rohbeck, Die Fortschrittstheorie der Aufklärung, Fráncfort y Nueva York 1987, 79 ss.; Christoph von Albrecht, Geopolitik und Geschichtsphilosophie 1748-1798, Berlín 1998.

32 Anne Robert Jacques Turgot, Über die Fortschritte des menschlichen Geistes, ed. Johannes Rohbeck y Lieselotte Steinbrügge, Fráncfort, 1990, 198.

33 Elke Uhl, "Gebrochene Zeit? Ungleichzeitigkeit als geschichtsphilosophisches Problem», en Rohbeck y Nagl-Docekal (eds.), op. cit, 50 ss. 
La simultaneidad de lo no-simultáneo no sigue siendo simplemente actual hoy, sino que ha experimentado una radicalización. El trasfondo de experiencia lo forman las corrientes migratorias (posibilitadas por las técnicas de transporte) de trabajadores, refugiados y turistas, que trabajan y conviven en las metrópolis. A esto hay que añadir la simultaneidad de las telecomunicaciones a través de la televisión y de internet. Como las técnicas modernas hacen posible la comunicación sincrónica, la simultaneidad de culturas no-simultáneas se puede experimentar hoy en cualquier lugar ${ }^{34}$. Este tipo de ampliación y condensación tiene como consecuencia que diferentes culturas del mundo se pueden vivir al mismo tiempo en un lugar ${ }^{35}$. La simultaneidad y la no-simultaneidad coinciden ahora en el espacio. La no-simultaneidad se ha incrementado hasta llegar a ser una no-simultaneidad perceptible espacialmente. De acuerdo con este concepto, yo denomino a este fenómeno la igualdad espacial de lo que es desigual espacialmente [Gleichräumlichkeit des Ungleichräumlichen].

¿Qué hemos ganado así? En mi opinión, dos aspectos con contenido que pueden tener consecuencias para la filosofía de la historia: la materialidad del espacio y la pluralidad de los espacios.

\section{La materialidad del espacio}

Ya en la historia de la filosofía el espacio se refiere a lo material, a la materialidad del mundo. Esta problemática se puede trasladar a la filosofía de la historia. Mientras se entienda la globalización como un proceso en el que las redes de información se van independizando, mientras se crea que la globalización sucede sobre todo o exclusivamente en internet, se tendrá la impresión de que el espacio ha desaparecido. Parece que ya sólo hay espacios virtuales, pertenecientes a un reino puramente espiritual de informaciones. Por tanto, no es una casualidad que las teorías sobre los medios de comunicación sean cada vez más «idealistas». Esta tendencia se percibe hasta en la filosofía: como si fuera un juego de ordenador, el mundo parece una mera construcción o interpretación. Contra esta ilusión se hacen notar cada vez más las condiciones materiales del espacio físico, especialmente en las ciencias de la comunicación ${ }^{36}$.

Así pues, el giro hacia el espacio significa que la filosofía de la historia se ha vuelto más realista, por no decir más materialista. El discurso se remateria-

${ }^{34}$ Niklas Luhmann, «Gleichzeitigkeit und Synchronisation», en id., Soziologische Aufklärung 5, Opladen, 1990, 95 ss.; William J.Mitchell, «Die Ökonomie der Präsenz», en Stefan Münker y Alexander Roesler (eds.), Mythos Internet, Fráncfort, 1997, 15 ss.

35 Anthony Giddens, Konsequenzen der Moderne, Fráncfort, 1995, 28-29; Ulrich Beck, Was ist Globalisierung?, Fráncfort, 1997, 178.

${ }^{36}$ Cfr. Alexander C. T. Geppert, Uffa Jensen y Jörn Weinhold (eds.), Raum und Kommunikation im 19. und 20. Jahrhundert, Bielefeld, 2005. 
liza, trata de los hechos espaciales y materiales, de la experiencia física de los espacios culturales: de la materialidad espacial de la historia. Las distancias espaciales vuelven a ser muy importantes. El lugar de la comunicación virtual lo ocupa ahora el tráfico material. De este modo, las condiciones climáticas y geográficas adquieren importancia. Para la historiografía esto significa prestar más atención a la economía, a la técnica y a la ecología. A la vista de la amenaza de catástrofes ecológicas, las condiciones naturales son cada vez más importantes. En este contexto se relativiza el concepto de desnaturalización del espacio histórico.

\section{La pluralidad de los espacios}

La diversidad de espacios culturales conduce a una comprensión plural del espacio. Por tanto, el spatial turn está relacionado estrechamente con el giro hacia la teoría de la cultura. Esto vale también para la filosofía de la historia: cuando se toman en consideración los espacios naturales y culturales de la historia, las diferencias del desarrollo cultural saltan más a la vista.

Este giro cultural se puede estudiar en el teorema ejemplar de la no-simultaneidad (o de la desigualdad espacial). El concepto de lo no-simultáneo presupone, al menos en los primeros tiempos, un desarrollo homogéneo. Cuando Turgot constata que pueblos de lugares diferentes se encuentran en el mismo nivel cultural en el que su propio pueblo se encontraba en el pasado, presupone la idea de progreso, de acuerdo con cuya escala hay que medir y ordenar las culturas de todo el mundo. Y cuando Turgot deriva la «desigualdad de las culturas» exclusivamente de la velocidad del progreso, confía en que los diferentes niveles se igualarán «con el paso del tiempo».

A este procedimiento se le ha reprochado con razón que es eurocentrista y cree en el progreso. La teoría de la no-simultaneidad se basa hasta el día de hoy en la idea de una evolución cultural que se consuma más o menos según el mismo esquema. De lo contrario no podríamos decir que una cultura determinada no se encuentra a la «altura de la época», en el nivel alcanzado hoy por la civilización. En este sentido se habla de «países en desarrollo» o de la «Europa de las dos velocidades»: igual que los trenes viajan sobre los mismos raíles a diferentes velocidades. Quien habla de no-simultaneidad se apoya en la teoría de la modernización siempre igual. Pero no ha habido ni hay un proceso homogéneo de aceleración general.

En este punto, el concepto de desigualdad espacial podría aportar un contenido nuevo. Ciertamente, la no-simultaneidad y la desigualdad espacial describen formalmente el mismo fenómeno, pero el concepto de desigualdad espacial subraya más la diversidad de las culturas; una diversidad que el esquema de desarrollo unitario impide observar y valorar. Con el espacio plural, el esquema temporal del proceso de civilización pasa a segundo plano, y 
con la pluralidad de los espacios los nuevos conflictos adquieren un significado mayor. El concepto de desigualdad espacial subraya particularmente el proceso de exclusión. Podemos hablar aquí de un espaciamiento de la desigualdad. De este modo renunciamos a la idea de un progreso uniforme y constatamos que el proceso de modernización no transcurre en línea recta y sin parar, sino que significa para algunas culturas rupturas y marginaciones.

\section{Globalización y cooperación}

No obstante, surge el problema de cómo pensar la actuación conjunta de los espacios históricos materializados y pluralizados. De la respuesta depende que la idea de historia universal y la concepción de la filosofía de la historia se puedan rehabilitar. Podemos ver en la filosofía de la historia del siglo XVIII una primera reflexión teórica acerca de esto ${ }^{37}$. El postulado de la primera Ilustración de la unidad e igualdad de todos los seres humanos es sustituido en la Ilustración posterior por la prueba histórica de las interacciones mundiales. La idea de humanidad no se limita a un punto de vista teórico, sino que se basa en las posibilidades de realización. La historia universal es la posibilidad real de una sociedad mundial.

Hoy los historiadores ven en la historia universal o mundial un enfoque legítimo y particular que, como disciplina parcial, estudia nexos de acción de gran extensión espacial. La meta es analizar relaciones interculturales y procesos de transferencia en el espacio histórico, como las migraciones, las conquistas, los movimientos de colonización y las transferencias de cultura. El regreso de lo geográfico desempeña aquí una función particular, y permite tematizar la cooperación global, pero también la amenaza ecológica. En este contexto surgió la idea de una nueva world history o global history ${ }^{38}$.

$\mathrm{Si}$ aceptamos esta historia mundial hecha realidad, tenemos que definir con más precisión el nexo de acción global ${ }^{39}$. Un acontecimiento forma parte de la historia mundial si, pese a haber sucedido en un lugar delimitado, tiene unos efectos que se derraman por todo el mundo histórico. Así, la industriali-

37 Herta Nagl-Docekal (ed.): Der Sinn des Historischen. Geschichtsphilosophische Debatten, Fráncfort, 1996; Heinz-Dieter Kittsteiner, Listen der Vernunft. Motive geschichtsphilosophischen Denkens, Fráncfort, 1998; Johannes Rohbeck, Technik - Kultur - Geschichte. Eine Rehabilitierung der Geschichtsphilosophie, Fráncfort, 2000; Geschichtsphilosophie zur Einführung, Hamburgo, Junius, 2004; Rohbeck y Nagl-Docekal (eds.), Geschichtsphilosophie und Kulturkritik, Darmstadt, 2003; Antonio Gómez Ramos, Reivindicación del centauro. Actualidad de la filosofia de la historia, Madrid, 2003; Peter Kosloswski (ed.), The Discovery of Historicity in German Idealism and Historism, Berlín, Heidelberg y Nueva York, 2005.

38 William H. McNeill, «A Defence of World History», en Transactions of the Royal Historical Society 32 (1982), 75 ss.

39 Johannes Rohbeck, «Weltgeschichte», en Historisches Wörterbuch der Philosophie, vol. 12, Basilea, Schwabe, 2005, 479 ss. 
zación surgió en Inglaterra y se extendió por todo el planeta, la Revolución Francesa afectó al desarrollo político de todo el mundo y la caída del muro de Berlín fue el punto de partida local del nuevo orden global. En este sentido, esos espacios han obtenido un sentido histórico universal.

También se puede incluir en la historia mundial un acontecimiento que posee no sólo muchos efectos fácticos, sino además mucha fuerza simbólica. Acontecimientos de este tipo han sido últimamente sobre todo las catástrofes que la humanidad entera ha percibido y recuerda gracias a los medios de comunicación, como el 11 de septiembre de 2001 en Nueva York, el atentado en Madrid de 2004 o el tsunami de ese mismo año en Asia. Estos acontecimientos provocaron una compasión colectiva. Esto recuerda a Kant, que vio en la Revolución Francesa un «signo histórico» porque suscitó en los contemporáneos un sentimiento moral de aprobación. La reacción interior de los observadores que se interesan a distancia por un acontecimiento es un indicio de la actuación moral futura. En el caso de la disposición fáctica a hacer donativos, esta actuación incluso se realizó a nivel mundial.

Además, es posible analizar la historia mundial como un nexo de cooperación global ${ }^{40}$. Filosóficamente se entiende por cooperación una actuación social en la que diferentes personas contribuyen a la realización de una meta compartida o común. Aquí hay que distinguir entre metas colectivas y comunes (por una parte) y convicciones compartidas y recíprocas (por otra parte), así como entre una actuación conjunta y una actuación colectiva. Por tanto, la diferencia consiste en una cooperación de individuos que mediante acciones individuales alcanzan una meta que es beneficiosa para todos los que la comparten posteriormente y una cooperación en la que un grupo de personas busca de antemano y colectivamente una meta. Así pues, la actuación colectiva presupone una meta común o una intención colectiva.

Si trasladamos estas definiciones abstractas a los sistemas sociales, podemos atribuir los dos tipos de cooperación a las dos esferas de economía y política. Mientras que en la economía la meta común del bienestar sólo se puede establecer de manera indirecta, en la política las metas colectivas se pueden buscar de manera directa. Este conflicto básico entre la sociedad y el Estado ha estado activo desde el principio de la Ilustración y la modernidad. En los Estados nacionales cerrados se podía al menos intentar conciliar estas tendencias opuestas. Pero en cuanto las empresas empiezan a actuar más allá de las fronteras nacionales, la política pierde su influencia. En esto consiste la crisis actual de la globalización. Su dimensión espacial consiste en que la economía se des-territorializa, mientras que la política sigue ligada a los viejos territorios.

40 Cfr. Tuomela Raimo, Cooperation. A Philosophical Study, Londres, Kluwer, 2000, 26 ss., 313-314; John R. Searle, The Construction of Social Reality, Londres, Pinguin Press, 1995, 23 ss.; Sylke Bartmann et al. (eds.), Kollektives Handeln. Politische Mobilisierung zwischen Struktur und Identität, Düsseldorf, 2002. 
Este problema fundamental es tematizado en el debate actual sobre la globalización. A esto se une la exigencia de recuperar el poder político sobre una economía mundial que está fuera de control. Cada vez es más intenso el clamor por unas organizaciones internacionales que tengan unas metas globales, como unas instituciones financieras internacionales que creen un orden financiero mundial. De las organizaciones ya existentes, como el Banco Mundial y la Organización Internacional del Comercio, se exige que no sólo propugnen la desregulación, sino que actúen políticamente en conformidad con las metas de Naciones Unidas. Ya que estas exigencias de «política mundial» se están empezando a realizar, se puede constatar ya un cambio en la globalización. De esta manera, la política y la actuación colectiva obtienen un espacio de acción global. 